Horizons philosophiques

\title{
Pourquoi l'existentialisme est-il né de penseurs religieux?
}

\section{Gabriel Malenfant}

Volume 16, numéro 2, printemps 2006

Héritage et réception de la pensée existentialiste

URI : https://id.erudit.org/iderudit/801316ar

DOI : https://doi.org/10.7202/801316ar

Aller au sommaire du numéro

\section{Éditeur(s)}

Collège Édouard-Montpetit

\section{ISSN}

1181-9227 (imprimé)

1920-2954 (numérique)

Découvrir la revue

\section{Citer cet article}

Malenfant, G. (2006). Pourquoi l'existentialisme est-il né de penseurs religieux? Horizons philosophiques, 16(2), 9-20. https://doi.org/10.7202/801316ar d'utilisation que vous pouvez consulter en ligne.

https://apropos.erudit.org/fr/usagers/politique-dutilisation/ 


\title{
Pourquoi l'existentialisme est-il né de penseurs religieux?
}

\author{
L'objet fondamental ou ultime de l'existentialisme est \\ l'individu existant, concret, vivant. Les existentialistes ne \\ sont pas intéressés, en premier lieu, par la vie humaine en \\ général, mais ils le sont plutôt par la vie et l'existence des \\ individus particuliers. Ils ont un intérêt pour la vie humaine \\ vue comme une série de décisions et de choix, et leur \\ premier but est de nous faire prendre conscience de nous- \\ mêmes en tant qu'individus vivants qui, en toute liberté, \\ prenons des décisions et en sommes responsables 12 .
}

L'existentialisme tel qu'on l'a connu au courant des trois premiers quarts du vingtième siècle ne dura qu'un moment infime de l'histoire de la philosophie. Pourtant, il la marqua profondément. Ses représentants ont écrit selon des styles divergeant les uns des autres; des derniers grands efforts de pensées systématiques jusqu'à la littérature romanesque, les penseurs existentialistes furent hétéroclites et il peut parfois sembler difficile de cerner ce qui, sans les unifier, les rassemble. Comme le décrit brièvement la citation mise en exergue, le lien de filiation entre les existentialistes serait une thématique commune à l'œuvre chez tous ces penseurs : l'existence humaine. S'il est vrai que de Merleau-Ponty à Derrida et de Sartre à Lévinas la vie de l'homme fut toujours au centre des préoccupations, il est de mon avis que l'existentialisme, malgré la pluralité de vues qui le composent, trouve aussi cohésion par le truchement d'un certain point d'origine, Søren Kierkegaard (1813-1855). D'une manière radicalement différente de celle de Kant ou de Hume, il a réussi à délimiter la raison humaine dans son rapport au monde et par lui, une nouvelle manière de poser les problèmes de la vérité et du vécu humain fut ainsi proposée.

Mais pourquoi l'existentialisme est-il né d'un penseur profondément croyant; d'un philosophe qui refusait d'en être un, refus empreint d'une ironie marquant la vanité des prétentions universalistes de ceux qui s'affublaient de ce titre eux-mêmes? La réponse à cette question se trouve d'après moi chez Hermann Cohen (1842-1918), philosophe juif allemand dont l'œuvre se trouve à cheval sur la fin d'un siècle et sur le début d'un autre; mais aussi à cheval sur deux philosophies : le postkantisme et la philosophie de la religion. Loin de moi l'idée 
d'argumenter que tous les existentialistes sont des penseurs religieux (il serait par exemple ridicule de tenter de tirer Albert Camus de ce côté). Plutôt, je me propose de considérer l'influence marquante qu'ont eue certains penseurs religieux sur le développement de l'existentialisme. Ainsi, je tirerai de Cohen une schématisation qui nous permettra de mieux cerner la question religieuse eu égard à la philosophie en général, et à l'existentialisme en particulier.

\section{La place de la religion au sein de la raison}

De quelle nature est le phénomène religieux et quelle place ce phénomène possède-t-il au sein de la raison? D'un point de vue historique, «le concept (de religion) est entièrement réduit au bilan de l'évolution ${ }^{3}$ ". Or, selon Cohen, le religieux représente plus qu'un simple phénomène culturel évolutif car il s'assoit sur certaines constantes dont la science historique ne peut rendre compte (notamment, le rapport intime de l'homme aux textes religieux). Ainsi, Cohen fera appel à la philosophie pour définir ce qu'est le religieux, car c'est en deçà de la science historique qu'il lui faut chercher pour trouver la source des concepts. La philosophie est, selon l'auteur, "la science de la Raison", elle-même vue comme "organe des concepts» à l'origine de toute connaissance : on aperçoit donc que c'est avec la naissance du cadre réflexif propre à la raison qu'émerge le religieux en tant que phénomène culturel. Par cette méthode anté-historique, Cohen circonscrit négativement ce qui, en faisant appel à la Raison comme fondement, doit être exclu de l'étude en question. La sensibilité, la passion et autres affects peuvent être écartés, puisque d'emblée, ils ne sont pas des sources possibles de la religion 4 . La raison serait alors, ipso facto, source de la religion, qui elle-même, à son tour, interpellerait la raison. De cette façon, Cohen coupe court à la tendance, présente depuis l'Aufklärung, à délaisser la religion au profit d'un savoir scientifique que l'on dit plus raisonnable. On peut conséquemment conclure de ceci que s'il existe un lien de nécessité entre religion et raison, alors : non seulement la source rationnelle de la religion est-elle un fait nécessaire, mais aussi, "(qu'il) ferait injure à la Raison de considérer que la religion ne serait qu'un domaine contingent de l'imagination ou de son rapport aux forces motrices des passions ${ }^{\text {" }}$. II montre plutôt que la religion n'est pas une sous-catégorie de connaissance qui serait dépassée par le progrès scientifique, mais qu'elle a au contraire grandi avec la science comme voisine. 
Mais malgré cette reconnaissance de la légitimité rationnelle du phénomène religieux, force est d'admettre que l'éthique, en tant que partie de la philosophie vue elle aussi comme discipline rationnelle, semble accaparer de façon exclusive l'ensemble du contenu théorique ayant trait aux problèmes humains dans leur spécificité morale ${ }^{6}$. En conséquence, il est légitime de se questionner quant au contenu cognitif dont peut disposer le domaine religieux, puisque : soit, d'une part, (1) la religion semble tomber sous le joug de l'éthique et perd toute autonomie; soit, (2) d'autre part, l'éthique se doit de partager son champ d'expertise avec la religion, ce qui impliquerait qu'il y ait deux méthodes rationnelles distinctes qui entreraient en concurrence.

Cohen optera pour une alternative différente des deux qu'il présente au début de Religion de la Raison tirée des sources du Judaïsme (1918). II faut comprendre que si la problématique d'un empiétement de l'éthique sur le religieux nous paraît insoluble, c'est que nous faisons une erreur herméneutique importante. L'éthique et le religieux ne peuvent entrer en compétition au sens où la première "n'est tout simplement pas en mesure de comprendre ni de reconnaître l'individu autrement que sous l'espèce d'humanité ${ }^{7}$ ". Malgré l'État qui vise - par le nationalisme ou par d'autres types de socialisation - à agir comme médiateur entre l'individu et l'humanité, il manque à l'éthique une perspective importante sur la réalité humaine; perspective dont elle ne peut rendre compte. En effet, alors que l'éthique s'acharne à déployer un universalisme des actions bonnes pour tous - ou à tout le moins, une méthode pour identifier celles-ci -, la religion tend plutôt à spécifier le rapport intime qui est vécu par les individus entre eux. En caractérisant les horizons théoriques de cette façon, nous pouvons concevoir que l'éthique et la religion ne s'usurpent pas mutuellement une place sous le soleil de la raison, mais qu'elles se complètent plutôt l'une et l'autre dans la mesure où la singularité de chaque individu est préservée par la religion, alors que seule la collectivité impersonnelle de ces individualités est considérée par l'éthique :

En s'opposant méthodiquement à tout ce qui est sensible et empirique chez l'homme, l'éthique en arrive à cette brutale conséquence qu'elle arrache d'emblée le moi humain à tout individualité pour la lui restituer, à un niveau plus élevé, sous une forme non seulement supérieure, mais aussi plus épurée. Ce moi de l'homme devient en elle le moi de l'humanité. (...) 
En tant que tel, il ne perd pas la caractéristique d'individualité en devenant le symbole de l'humanité; c'est uniquement l'humanité qui lui confère, à travers ce symbole, une véritable individualité8.

Le problème est donc le suivant : tant et aussi longtemps que l'homme ne fait pas un effort d'abstraction qui lui permette d'échapper à son contexte empirique, social et sensible, il ne fait pas partie de cette «humanité» épurée de travers charnels dont se soucie l'éthique. L'homme éthique en est un à qui on refuse le droit d'agir selon des comportements fondamentalement humains au profit soit d'un calcul de conséquence, soit de l'application d'une maxime déontologique. L'homme, sous la seule lorgnette éthique, n'est plus incarné, mais éthicisé, assujetti à un idéal rationnel qui le dépasse. En conséquence, les éthiciens n'ont pu explorer la possibilité de considérer l'homme autrement qu'en tant que représentant universel - et ceci est vrai tant d'un point de vue conséquentialiste que déontologique -, ce qui a eu pour effet de contrecarrer toute véritable élaboration d'une philosophie de l'existence jusqu'à l'époque de Cohen. Bien que plusieurs penseurs des XVIIIe et XIXe siècles aient pu concevoir le statut de l'éthique de cette façon (comme Kierkegaard, justement), Cohen fut le premier à définir aussi clairement son objet, son utilité, mais surtout, sés limites. La réalité humaine serait donc toujours plus complexe que toute théorie éthique possible, et ce, aussi complexe que cette dernière puisse être, simplement parce que l'éthique est un idéal rationaliste par nature et que le rationalisme évacue un nombre important de réalités de la vie humaine.

Ce qu'affirme Cohen, c'est en fait que la concrétude et la richesse de la relation avec autrui dépassent et dépasseront toujours le pouvoir limité de la raison et que, par conséquent, l'idéal éthique ne peut constituer le seul discours valable quant aux relations des hommes avec ceux qui les entourent. C'est en ce sens que la religion occupe une place qui lui est propre au cœur de la raison. Et, comme nous le verrons, c'est aussi en ce sens que l'existentialisme est tributaire de penseurs religieux; admettre que l'existence humaine dépasse le domaine du sensible ou du rationnel, c'est enfin comprendre l'homme pour ce qu'il est - un être à la fois sensible, émotif, et rationnel - et non pour ce que l'on voudrait qu'il soit - un être gouverné par le seul pouvoir de sa raison. 


\section{Du rapport à l'«autre homme» au rapport à «autrui»}

En suivant Cohen, on remarque que l'éthique est impersonnelle de par la méthode rationnelle qu'elle utilise. Sur le plan langagier, elle ne s'intéresse qu'au «il» et au «je», tous deux vus sous un angle neutre et détaché de toute corporéité. A contrario, la religion s'intéresse au «tu», modalité langagière qui marque directement ma relation avec l'autre et insuffle de ce fait une personnalité à l'alter ego dont l'existence, dans le contexte d'une éthique universaliste telle que décrite par Cohen, est réduite à un «il» n'ayant aucune prise sur la réalité relationnelle. Pourtant, dans une relation dialogique avec autrui, le tiers est toujours exclu. Dans l'exemple que Cohen donne du stoïcisme en lien avec la prise en compte de la souffrance humaine ${ }^{8}$, il démontre que c'est par l'attention portée aux conditions empiriques des individus que la religion donne vie au «il»; malgré le fait que la méthode intellectuelle demeure éthique, l'alter ego ne peut devenir un "tu» pour moi que dans la mesure où sa réalité empirique est tenue pour importante, ce à quoi se refuse l'éthique pour des motifs structurels. Dans les différentes doctrines déontologiques, ces motifs sont évidents puisque cette éthique repose entièrement sur des principes établis par la raison (impératif catégorique, Kant). Mais bien que le conséquentialisme prenne en compte la réalité empirique, il tombe néanmoins sous la critique de Cohen. C'est que même si, pour John Stuart Mill, l'utilitarisme est vu comme concret puisqu'il repose sur des observations empiriques, il reste qu'un "calcul», qu'il soit de conséquences ou autre, est toujours une mise en relation d'entités abstraites. Or, même si ces abstractions sont tirées de faits concrets, le calcul demeure idéel et vise d'entrée de jeu l'universalisation; d'où la critique cohenienne de l'éthique en général, et non pas d'une éthique (déontologique, par exemple) en particulier. L'éthique de la vertu suggère d'ailleurs elle aussi de fortes balises rationnelles pour évaluer nos actions. Dans tous les cas, la réalité factuelle est reléguée au second rang par l'éthique, derrière une conceptualisation qui rationalise l'application de comportements jugés adéquats a priori (ou, si a posteriori, reposant inévitablement sur d'importantes abstractions faisant fi de la réalité particulière et charnelle de l'homme) en dépit d'une réalité effective qui dépasse à tout coup cette rationalisation. En effet, comment calculer le poids de la souffrance d'autrui dans un raisonnement éthique lorsque son visage meurtri me heurte de plein fouet? Et même s'il était possible d'y arriver : comment, par la suite, être en mesure d'intégrer cette variable à un calcul visant 
l'accroissement du bien-être général alors que celui qui souffre se trouve face à moi, m'interpellant directement pour obtenir de l'aide? Encore une fois, nous voyons que l'éthique est d'une aide précieuse lors de l'élaboration de règles générales, mais que la réalité factuelle dépasse souvent ce qui peut être considéré par l'éthique, et ce, parce que le domaine d'application de l'éthique - le monde - ne frappe pas que notre seule raison, mais plutôt notre être entier selon ses multiples facettes.

Toutefois, la prise en compte de la souffrance de l'autre, par la pitié notamment, ne signifie pas que je doive m'y abandonner ou m'y perdre aveuglément. Au contraire, dans une perspective commune à l'éthique, la logique religieuse permet à l'individu de choisir, ou à tout le moins, de «mettre en question l'ensemble de (son) orientation dans le monde moral 10 " et d'opter ainsi pour une véritable connaissance de soi-même et de l'autre en dehors des limites théoriques imposées par le raisonnement éthique. En cela, Cohen est clairement précurseur de Buber, de Rosenzweig et de Lévinas (nous comprendrons mieux pourquoi plus loin). Mais, par suite, nous devons saisir que le concept d'homme ne signifie pas la même chose qu'il soit considéré d'un point de vue éthique ou d'un point de vue religieux. Dans le premier cas, il se réfère à l'Homme sous le joug de l'humanité ou sous celui d'une entité universelle, alors que dans le second cas, l'homme est un individu particulier, avec une enveloppe charnelle qui constitue sa réalité quotidienne. Selon Cohen, en conséquence, il est clair que la normativité aride de l'éthique doit être à tout le moins complémentarisée par la souplesse morale de la religion. Or, d'après lui, c'est aussi par Dieu que la religion se distingue de l'éthique. Pourtant, il admet que l'éthique a souvent assimilé Dieu à ses systèmes. Mais il faut, comme nous l'avons fait pour le concept d'homme, distinguer le Dieu éthique du Dieu religieux : alors que le premier est simplement le garant de la moralité universelle ou de la possibilité de la vérité (Kant, Descartes, etc.), le Dieu religieux est, par opposition, le Dieu des pauvres, des indigents, de la souffrance humaine ${ }^{11}$. Les sources juives confirment en effet qu' 'Ezékiel a transmis à la religion le Dieu de l'individu singulier12". Selon ces enseignements, nous pourrions affirmer avec Cohen que, contrairement à la croyance courante de notre époque, l'éthique guide, alors que la religion concrétise.

Ainsi, Cohen affirme un primat logique de la religion par rapport à l'éthique; comme il le dit lui-même, le «tu» de la deuxième personne précède le «il» de la troisième ${ }^{13}$. Martin Buber reprendra presque 
intégralement cette caractérisation des pronoms comme indicateur de relations particulières entre les hommes, le monde et Dieu; et comme Cohen, Buber refusera de ne voir, dans ce rapport religieux primordial, qu'une possibilité pour l'«homme au singulier» kierkegaardien. Avec Buber, Gaston Bachelard affirmera en préface de Je et Tu que de "dire avec Kierkegaard que la foule est fausseté, c'est manquer au devoir de substituer ce qui est à ce qui doit être ${ }^{14}$ ». L'idée maîtresse serait donc de "démêler la mêlée» par le "tu» pour individualiser la multitude informe de la masse, et enfin, de l'humanité. C'est justement ce que permet la religion. Pour guider, l'éthique doit avoir un pied dans l'empirie malgré son désir de demeurer purement rationnelle, et conséquemment, elle doit aussi avoir un pied dans la religion; au contraire, le religieux n'a pas besoin de l'éthique pour demeurer près de son objet, soit l'homme compris comme autrui.La souffrance n'accède à une valeur éthique que si elle est souffrance sociale. (...)[Or,] la souffrance est un sentiment actuel qui n'est pas simplement un fait social reflétant la misère, c'est un fait de première grandeur au sein de la conscience, et qu'il faut saisir et comprendre comme ce fait qui envahit la conscience et en détermine du même coup tous les autres processus, toutes les autres activités ${ }^{15}$.

L'alter ego devient ainsi, par ma rencontre avec lui, autrui. À la souffrance que je décèle chez autrui, je ne peux rester indifférent : "par le biais de la souffrance et de la pitié, le 'tu' a été révélé dans l'homme [et] le 'je', libéré des ténèbres de l'égoïsme ${ }^{16}$ ». Sur ce visage qui m'est présenté à nu — dans ce face à face impudique, dirait Lévinas - une responsabilité me frappe; “dire Tu, c'est dire en même temps le Je du couple verbal Je-Tu17». Or, pour l'éthique, ce visage souffrant n'est que celui d'un drone, et le mien aussi d'ailleurs; et cet automate ne répond plus qu'à des règles qu'on lui a inculquées, ne répond plus qu'à une espèce élevée au rang d'idéal : l'humanité - le voilà l'homme éthicisé! II répond, certes, mais parce qu'il connaît une règle qui lui commande de le faire, pas parce qu'il est touché, ni parce qu'il est cet homme-là, cet individu unique et singulier qui interagit avec autrui. Plutôt, il répond parce qu'il est un homme - un homme parmi tant d'autres - qui se perd dans une mer d'cil(s)", et que les «il(s)» flottent isolés dans un océan de règles immuables, dans une réelle déréliction. Lorsque la réalité dépasse le domaine de l'application prévue par la règle, l'homme éthicisé est perdu, sans repère seul dans son «il». À raison trop assurée, on n'assure que sa solitude. 


\section{L'existentialisme et la concrétude du phénomène religieux}

Le concept logique primordial, qui se trouve partout sous-jacent dans l'œuvre finale de Cohen, est celui de corrélation. Par ce concept, il met en lumière les relations qu'entretiennent, en premier lieu, l'homme avec Dieu, puis en second lieu, l'homme avec l'autre homme. Comme nous l'avons vu, la relation avec l'autre homme en est une qui repose sur la prise en compte de l'individu lui-même, et non de son appartenance à une entité plus grande (que celle-ci soit une catégorie d'espèce ou de nationalité). Or, selon Cohen, cette "relationalité" repose à son tour sur la corrélation première que nous entretenons avec Dieu. Cette relation est intime et singulière, mais surtout, n'est pas unidirectionnelle. Pour plusieurs penseurs religieux, le Dieu infini donne tout à l'homme qui lui, n'est au fond que finitude radicale - d'où l'unidirectionalité du vecteur Dieu-homme. Pourtant, Cohen raffine la logique religieuse en se saisissant d'un concept logique issu de la tradition juive qui influencera grandement l'existentialisme et par conséquent, l'histoire de la philosophie. Du point de vue de l'homme, "ce n'est pas le monde de l'expérience qui est, comme le veut l'empirisme, le fondement de la connaissance; c'est, au contraire, le monde de la connaissance qui fonde le monde de l'expérience 18 ». L'essentiel de ce constat est kantien et correspond à la fameuse "révolution copernicienne» de l'illustre philosophe, mais Cohen poussera l'idée plus loin. C'est que l'acceptation de ce constat le mène inévitablement à l'idée de corrélation puisque "(l)a connaissance de Dieu, et en ce sens l'existence de Dieu pour nous, dépend (donc) de notre rationalité; inversement, notre rationalité ainsi que notre existence dépendent de la relation que nous avons avec Dieu en tant qu'il est notre créateur ${ }^{19}$ ». Par conséquent, il est de ce fait impossible de savoir ce qui précède quoi à l'intérieur de cette logique puisque poser la question d'une origine, cela revient à privilégier un point de vue inexistant; seule existe la relation. Plus important encore, cette corrélation existe parce que les deux termes présents (l'homme et Dieu) sont les deux pôles d'un rapport qui préexiste à leur séparation logique; en ce sens "phénoménologique", l'existence de Dieu est logiquement dépendante de notre rationalité.

Mais à ce stade de la démonstration, le lecteur peut légitimement se demander : "Qu'est-ce que Dieu a à voir avec l'existentialisme si, justement, ce courant est nommé ainsi grâce à son intérêt pour l'existence finie et particulière de l'homme»? En fait, il faut comprendre que pour arriver à traiter de l'existence humaine en demeurant près de sa 
réalité, il a fallu laisser de côté certains pans importants de la philosophie traditionnelle; surtout, l'effort constant de systématicité, de complétude des systèmes et de recherche d'un absolu par la seule raison ${ }^{20}$. Avec le Cohen de La religion dans les limites de la philosophie (1915) et de Religion de la raison (1918) on assiste à une mise en forme claire d'une problématique fondamentale de l'existentialisme, soit la trop grande place de la raison pure dans l'appréhension de l'homme et la substitution de cette omnipotence de la raison en éthique par une rationalité différente visant à comprendre l'homme incarné et vivant avec d'autres hommes avec lesquels il entretient des relations primordiales et dépassant qualitativement le cadre rationnel traditionnel21. C'est aussi avec lui qu'on assiste à la réhabilitation d'une manière logique de concevoir le monde qui avait depuis longtemps été ignorée ou reléguée au second plan par les penseurs les plus influents de la tradition philosophique, soit, la logique corrélative issue de la tradition judaïque. Par conséquent, grâce à ces ouvertures philosophiques importantes et à un langage qui fut repris par plusieurs (depuis le Je et le Tu, tel que susmentionné), on peut affirmer que l'influence de Cohen et de la pensée religieuse sur l'existentialisme est indéniable et sous-estimée. Ce n'est donc pas tant l'idée de Dieu, ni le concept de religion d'ailleurs, qui sont importants pour l'influence de Cohen sur la tradition existentialiste - au contraire : il s'agit plutôt de l'explication que Cohen donne de la relationalité que l'homme entretient avec ce qui l'entoure (Dieu, autrui, le monde, la connaissance) qui compte pour comprendre le trait d'union qui le relie à la pensée existentialiste. Au sujet de la philosophie de Rosenzweig, qui fut l'élève et héritier direct de Cohen, Lévinas dit :

En moi - la totalité se brise. Rosenzweig nous habitue à penser le non synthétisable, la différence, contrairement à une tradition philosophique où le Même absorbe l'Autre dans son intériorité et où la pensée absolue est une pensée pensant l'identité du Même avec l'Autre.(...) Chez Rosenzweig, on entrevoit la 'positivité' d'un pluralisme absolu.

(...) II n'y aurait pas de synthèse posant Dieu côte à côte et le monde comme deux êtres coexistants avant la Création, toujours déjà passée, déjà précédant la conjonction qui unit des étants et qui, par conséquent, n'est concrète que dans cette Création; il n'y aurait pas de synthèse thématisable, telle 
que "Dieu et l'homme", sinon comme révélation où l'homme est interpellé dans toute l'acuité et dans toute l'actualité du maintenant - relation 'frontale' avant toute juxtapositionmaintenant où le passé lui-même ne s'affirme que comme re-présenté et ainsi comme "éternellement présent»; la conjonction et de la formule «l'homme et le monde» ne serait concrète que dans la réponse de l'homme à la Révélation et à l'amour de Dieu, dans l'ouverture sur le monde comme monde-à-venir et où, à sa façon, ce monde est «éternellement présent»; ouverture sur le monde qui n'est pas le désormais fameux être-au-monde, mais, d'emblée, rapport au monde futur ou monde meilleur ou monde à "améliorer" et, notamment, rapport aux autres hommes "personnages du monde", aux «ils» auxquels on apprendra à dire «tu». La Rédemption, dira Rosenzweig, c'est apprendre à dire «tu» à un «il»»22.

L'influence de Cohen sur Rosenzweig est ici marquée au fer rouge par Lévinas sans même qu'il ait à le nommer; l'éthique, à ne pas entendre au sens traditionnel chez Lévinas, devient philosophie première parce que l'existence humaine est relationnelle avant même que l'on ne soit en mesure de séparer les termes de cette relation en une pluralité d' 'individus" distincts. Chez lui se trouve donc à l'œuvre la logique corrélative que Cohen s'est efforcé de mettre en lumière. Mais l'influence de la religion telle que dépeinte par Cohen sur la pensée existentialiste ne se limite pas qu'à Buber, Rosenzweig et Lévinas. Elle déborde jusqu'à Merleau-Ponty qui, par sa phénoménologie, propose une nouvelle manière concrète de considérer la réalité humaine 23 ; elle déborde aussi jusqu'à Derrida, penseur des différences et de la différance, qui considère la relation comme ayant un primat sur les termes composant celle-ci au sein d'un système; et elle déborde même jusqu'à Sartre lorsqu'il affirme que «l'existence précède l'essence». Bref, la portée de l'argumentation cohenienne est impressionnante et dépasse le seul cadre de la pensée religieuse.

Ce n'est donc pas tant la religion - au sens des multiples religions et des systèmes de croyances - qui a influencé l'existentialisme, mais plutôt, la logique se trouvant derrière la pensée religieuse. Les existentialistes de tout acabit ne sont pas tous des penseurs religieux, loin de là, mais l'algèbre de leurs écrits reste néanmoins imprégnée des préoccupations et conceptions venues de 
la religion. Ce que les existentialistes ont montré, c'est donc qu'il n'y a pas d'obligation d'être pieux, ou même croyant, pour comprendre le but existentialiste visé par la religion. Le phénomène religieux a une logique qui lui est propre et qui peut être utilisée à l'extérieur des bornes prescrites par les textes sacrés. En cela, les existentialistes sont allés plus loin que ce que Cohen l'avait anticipé; mais il demeure que le fondement religieux de l'existentialisme ne peut être repoussé du revers de la main. La prépondérance des thèmes et de la logique de Cohen est indubitable dans l'existentialisme - qu'on revienne, pour appuyer ceci, à la citation de départ qui définissait l'existentialisme comme étant une philosophie non seulement intéressée par «l'individu existant, concret (et) vivant», mais aussi par "la vie humaine vue comme une série de décisions et de choix" desquels nous sommes responsables. La constante redéfinition de l'orientation morale de l'homme que permet la religion telle que vue par Cohen face à la prégnance d'un «tu» pour un «je» marque cette libre décision de l'homme toujours déjà en corrélation avec un autrui qui peut, par son agir - tant dialogique que non-dialogique - , faire un choix. Et ce choix, c'est l'ouverture que permet l'existentialisme.

\section{Gabriel Malenfant Philosophie, Université de Montréal}

1. II m'est essentiel de remercier Bettina G. Bergo (Université de Montréal) pour son soutien constant et Dominic Desroches (Collège de Rosemont) pour ses commentaires.

2. "(T)he fundamental or ultimate subject matter of existentialism is the existing, concrete, living individual. Existentialists are not primarily concerned with human life. in general, but with the life of particular existing individuals. They are concerned with human life viewed as a series of decisions and choices, and their first goal is to make us aware of ourselves as living individuals who, in our freedom, make decisions and are responsible for them". L. Nathan Oaklander, Existentialist Philosophy, An Introduction, Second Edition, Prentice Hall / Simon \& Schuster, New Jersey, 1996, p. 3. Ma traduction.

3. Hermann Cohen, Religion de la Raison tirée des sources du judaïsme, trad. Marc B. de Launay et Anne Lagny, Paris, PUF-Questions, 1994, p. 13.

4. Notamment, comme l'explique Cohen lui-même, parce que la religion est un phénomène typiquement humain, et que les caractéristiques énumérées ci-haut sont aussi possédées par les animaux.

5. C'est d'où est dérivé le titre de la section 6 de Religion de la Raison tirée des sources du judaïsme : "La religion de la Raison fait de la religion une fonction universelle de la conscience» (Hermann Cohen, op. cit., p.19.).

6. Il est important de voir que pour Cohen, la religion se distingue de la morale comme de l'éthique, ces deux dernières se trouvant subsumées sous le même idéal d' "éducation par lui-même du genre humain» (Hermann Cohen, op. cit., p. 158.). La religion est cette incamation de l'éthique qui s'actualise dans le monde et entre les 
hommes, mais d'abord entre eux et Dieu de manière primordiale. Nous y reviendrons.

7. Ibid., p. 28.

8. Ibid., p. 27-28.

9. Ibid., p. 32-34.

10. Ibid., p. 35.

11. Ibid., p. 38-41.

12. Ibid., p. 39.

13. Ibid., p. 29.

14. Martin Buber, Je et Tu, Paris, Aubier - Bibliothèque philosophique, 1969, c1923, p. 14 (préface de Gaston Bachelard).

15. Hermann Cohen, op. cit., p. 193-194.

16. Ibid., p. 36.

17. Martin Buber, op. cit., p. 20. Pour bien comprendre les reproches lévinassiens concernant Buber et ainsi voir à la fois l'influence et les divergences entre Lévinas et Buber, on pourra se référer au Totalité et Infini de Lévinas lui-même, mais aussi à «Violence et métaphysique» (in L'écriture et la différence, Éditions du Seuil, 1979), où Jacques Derrida, à la note de la page 156, analyse brièvement les distinctions du jeu des pronoms chez Buber et Lévinas.

18. Sylvain Zac, La philosophie religieuse de Hermann Cohen, Paris, Librairie Philosophique J. Vrin, 1984, p. 22

19. "Knowledge of God, and in that sense God's existence for us, depends on our rationality; conversely, our rationality and existence depend on the relationship we have to God as our creators. Robert Gibbs, Correlations in Rosenzweig and Levinas, New Jersey, Princeton University Press, 1992, p. 19. Ma traduction.

20. Par exemple, «le souci de Franz Rosenzweig, dès les premiers mots des écrits de L'Étoile, est de montrer comment toute totalité, tout effort de totalisation même est voué à l'échec, ou plutôt est impossible». Stéphane Habib, Lévinas et Rosenzweig: Philosophies de la Révélation, Paris, PUF/ Philosophie d'aujourd'hui, 2005, p. 21. On peut aussi noter déjà chez Husserl - dans L'idée de la phénoménologie (1907) et même avant, dans ses Recherches logiques (1900-1901) - ce rejet de la volonté de totalisation d'un Hegel au profit d'une tâche infinie, soit celle de la phénoménologie non pas de l'Esprit, mais plutôt celle de l'omniprésence de l'intentionnalité. Mais tisser un lien entre Cohen et Husserl demanderait un tout autre travail que nous ne ferons pas ici.

21. Le statut de ce constat est difficile à cerner; Cohen ayant été post-kantien tout au long de sa vie, il est singulier de voir ce schisme apparent avec le reste de sa philosophie qui, pour certains commentateurs comme Carole Prompsy et Marc de Launay, n'en est pas un. Nous ne prendrons pas clairement position ici, bien qu'il est de mise d'affirmer que si le fait de nommer cette nouvelle pensée un schisme par rapport au reste de l'œuvre de Cohen est peut-être trop dire, on doit mentionner, à tout le moins, la particularité du geste cohenien tardif au sein de son corpus.

22. Tiré de l'introduction faite par Lévinas au livre de Stéphane Mosès, Système et Révélation, Paris, Éditions du Seuil, 1982, p. 13-15.

23. "L'intérieur et l'extérieur sont inséparables. Le monde est tout au-dedans et je suis tout hors de moi." (Maurice Merleau-Ponty, "Situation du sujet», Existence et dialectique, Paris, PUF-SUP, 1971, p. 73.) Voilà une autre façon de traiter de la corrélation telle que reprise de Cohen par Buber. 